\title{
Distribution of Points of Interpolation and of Zeros of Exactly Maximally Convergent Multipoint Padé Approximants
}

\author{
R. K. Kovacheva \\ Institute of Mathematics and Informatics, Bulgarian Academy of Sciences, Sofia, Bulgaria \\ Email: rkovach@math.bas.bg
}

Received 20 March 2015; accepted 30 April 2015; published 5 May 2015

Copyright ( 2015 by author and Scientific Research Publishing Inc.

This work is licensed under the Creative Commons Attribution International License (CC BY).

http://creativecommons.org/licenses/by/4.0/

(c) (i) Open Access

\section{Abstract}

Given a regular compact set $E$ in $\mathbb{C}$, a unit measure $\mu$ supported by $\partial E$, a triangular point set $\beta:=\left\{\left\{\beta_{n, k}\right\}_{k=1}^{n}\right\}_{n=1}^{\infty}, \beta \subset \partial E$ and a function $f$, holomorphic on $E$, let $\pi_{n, m}^{\beta, f}$ be the associated multipoint $\beta$-Padé approximant of order $(n, m)$. We show that if the sequence $\pi_{n, m}^{\beta, f}, n \in \Lambda$, $\Lambda \subseteq \mathbb{N}, m$-fixed, converges exactly $\mu$-maximally to $f$ with respect to the $m$-meromorphy, then the points $\beta_{n, k}$ are uniformly distributed on $\partial E$ with respect to $\mu$ as $n \in \Lambda$. Furthermore, a result about the behavior of the zeros of the exact maximally convergent sequence $\Lambda$ is provided, under the condition that $\Lambda$ is "dense enough".

\section{Keywords}

Multipoint Padé Approximants, Maximal Convergence, Domain of $\boldsymbol{m}$-Meromorphy

\section{Introduction}

We first introduce some needed notations.

Let $\Pi_{n}, n \in \mathbb{N}$ be the class of the polynomials of degree $\leq n$ and $\mathcal{R}_{n, m}:=\left\{r=p / q, p \in \Pi_{n}, q \in \Pi_{m}, q \neq 0\right\}$.

Given a compact set $E$, we say that $E$ is regular, if the unbounded component of the complement $E^{c}:=\overline{\mathbb{C}} \backslash E$ is solvable with respect to Dirichlet problem. We will assume throughout the paper that $E$ possesses a connected complement $E^{c}$. In what follows, we will be working with the max-norm $\|\cdots\|_{E}$ on $E$, that is $\|\cdots\|_{E}:=\max _{z \in E}|\cdots|(z)$. 
Let $\mathcal{B}(E)$ be the class of the unit measures supported on $E$, that is $\operatorname{supp}(\cdots) \subseteq E$. We say that the infinite sequence of Borel measures $\left\{\mu_{n}\right\} \in \mathcal{B}(E)$ converges in the weak topology to a measure $\mu$ and write $\mu_{n} \rightarrow \mu$, if

$$
\int g(t) \mathrm{d} \mu_{n} \rightarrow \int g(t) \mathrm{d} \mu
$$

for every function $g$ continuous on $E$. We associate with a measure $\mu \in \mathcal{B}(E)$, the logarithmic potential $U^{\mu}(z)$, that is,

$$
U^{\mu}(z):=\int \log \frac{1}{|z-t|} \mathrm{d} \mu .
$$

Recall that $U^{\mu}([1])$ is a function superharmonic in $\mathbb{C}$, subharmonic in $\overline{\mathbb{C}} \backslash \operatorname{supp}(\mu)$, harmonic in $\mathbb{C} \backslash \operatorname{supp}(\mu)$ and

$$
U^{\mu}(z)=\ln \frac{1}{|z|}+o(1), \quad z \rightarrow \infty .
$$

We also note the following basic fact ([2]):

Carleson's lemma: Given the measures $\mu_{1}, \mu_{2}$ supported by $\partial E$, suppose that $U^{\mu_{1}}(z)=U^{\mu_{2}}(z)$ for every $z \notin E$. Then, $\mu_{1}=\mu_{2}$.

Finally, we associate with a polynomial $p \in \Pi_{n}$, the normalized counting measure $\mu_{p}$ of $p$, that is

$$
\mu_{p}(F):=\frac{\text { number of zeros of } p \text { on } F}{\operatorname{deg} p},
$$

where $F$ is a point set in $\mathbb{C}$.

Given a domain $B \subset \mathbb{C}$, a function $g$ and a number $m \in \mathbb{N}$, we say that $g$ is $m$-meromorphic in $B$ $\left(g \in \mathcal{M}_{m}(B)\right)$ if $g$ has no more than $m$ poles in $B$ (poles are counted with their multiplicities). We say that a function $f$ is holomorphic on the compactum $E$ and write $f \in \mathcal{A}(E)$, if it is holomorphic in some open neighborhood of $E$.

Let $\beta$ be an infinite triangular table of points, $\beta:=\left\{\left\{\beta_{n, k}\right\}_{k=1}^{n}\right\}_{n=1,2, \cdots}, \beta_{n, k} \in E$, with no limit points outside $E$ (we write $\beta \in E$ ). Set

$$
\omega_{n}(z):=\prod_{k=1}^{n}\left(z-\beta_{n, k}\right) .
$$

Let $f \in \mathcal{A}(E)$ and $(n, m)$ be a fixed pair of nonnegative integers. The rational function $\pi_{n, m}^{\beta, f}:=p / q$ where the polynomials $p \in \Pi_{n}$ and $q \in \Pi_{m}$ are such that

$$
\frac{f q-p}{\omega_{n+m+1}} \in \mathcal{A}(E)
$$

is called a $\beta$-multipoint Padé approximant of $f$ of order $(n, m)$. As is well known, the function $\pi_{n, m}^{\beta, f}$ always exists and is unique [3] [4]. In the particular case when $\beta \equiv 0$, the multipoint Padé approximant $\pi_{n, m}^{\beta, f}$ coincides with the classical Padé approximant $\pi_{n, m}^{f}$ of order $(n, m) \quad([5])$.

Set

$$
\pi_{n, m}^{\beta, f}:=\frac{P_{n, m}^{\beta, f}}{Q_{n, m}^{\beta, f}},
$$

where the polynomials $P_{n, m}^{\beta, f}$ and $Q_{n, m}^{\beta, f}$ do not have common divisors. The zeros of $Q_{n, m}^{\beta, f}$ are called free zeros of $\pi_{n, m}^{\beta, f} ; \operatorname{deg} Q_{n, m} \leq m$.

We say that the points $\beta_{n, k}$ are uniformly distributed relatively to the measure $\mu$, if

$$
\mu_{\omega_{n}} \rightarrow \mu, \quad n \rightarrow \infty .
$$

We recall the notion of $m_{1}$-Hausdorff measure (cf. [6]). For $\Omega \subset \mathbb{C}$, we set 


$$
m_{1}(\Omega):=\inf \left\{\sum_{v}\left|V_{v}\right|\right\}
$$

where the infimum is taken over all coverings $\left\{\sum V_{v}\right\}$ of $\Omega$ by disks and $\left|V_{v}\right|$ is the radius of the disk $V_{v}$.

Let $D$ be a domain in $\mathbb{C}$ and $\varphi$ a function defined in $D$ with values in $\overline{\mathbb{C}}$. A sequence of functions $\left\{\varphi_{n}\right\}$, meromorphic in $D$, is said to converge to a function $\varphi \quad m_{1}$-almost uniformly inside $D$ if for any compact subset $K \subset D$ and every $\varepsilon>0$ there exists a set $K_{\varepsilon} \subset K$ such that $m_{1}\left(K \backslash K_{\varepsilon}\right)<\varepsilon$ and the sequence $\left\{\varphi_{n}\right\}$ converges uniformly to $\varphi$ on $K_{\varepsilon}$.

For $\mu \in \mathcal{B}(E)$, define

$$
\rho_{\min }:=\inf _{z \in E} \mathrm{e}^{-U^{\mu}(z)}
$$

and

$$
\varrho_{\max }:=\max _{z \in E} \mathrm{e}^{-U^{\mu}(z)} ;
$$

( $U^{\mu}$ is superharmonic on $E$; hence, it attains its minimum (on $\left.E\right)$ ). As is known ([1] [7]),

$$
\mathrm{e}^{-U^{\mu}(z)} \geq \rho_{\text {min }}, \quad z \in E^{c},
$$

Set, for $r>\rho_{\min }$,

$$
E_{\mu}(r):=\left\{z \in \mathbb{C}, \mathrm{e}^{-U^{\mu}(z)}<r\right\}
$$

Because of the upper semicontinuity of the function $\chi(z):=\mathrm{e}^{-U^{\mu}(z)}$, the set $E_{\mu}(r)$ is open; clearly $E_{\mu}\left(r_{1}\right) \subset E_{\mu}\left(r_{2}\right)$ if $r_{1} \leq r_{2}$ and $E_{\mu}(r) \supset E$ if $r>\varrho_{\max }$.

Let $f \in \mathcal{A}(E)$ and $m \in \mathbb{N}$ be fixed. Let $R_{m, \mu}(f)=R_{m, \mu}$ and $D_{m, \mu}(f)=D_{m, \mu}:=E_{\mu}\left(R_{m, \mu}\right)$ denote, respectively, the radius and domain of m-meromorphy with respect to $\mu$, that is

$$
R_{m, \mu}:=\sup \left\{r, f \in \mathcal{M}_{m}\left(E_{\mu}(r)\right)\right\}
$$

Furthermore, we introduce the notion of a $\mu$-maximal convergence to $f$ with respect to the m-meromorphy of a sequence of rational functions $\left\{r_{n, v}\right\}$ (a $\mu$-maximal convergence), that is, for any $\varepsilon>0$ and each compact set $K \subset D_{m}$, there exists a set $K_{\varepsilon} \subset K$ such that $m_{1}\left(K \backslash K_{\varepsilon}\right)<\varepsilon$ and

$$
\limsup _{n+v \rightarrow \infty}\left\|f-r_{n, v}\right\|_{K_{\varepsilon}}^{1 / n} \leq \frac{\left\|\mathrm{e}^{-U^{\mu}}\right\|_{K}}{R_{m, \mu}(f)} .
$$

Hernandez and Calle Ysern proved the followings:

Theorem A [8]: Let $E, \mu, \beta$ and $\omega_{n}, n=1,2, \cdots$ be defined as above. Suppose that $\mu_{\omega_{n}} \rightarrow \mu$ as $n \rightarrow \infty$ and $f \in \mathcal{A}(E)$. Then, for each fixed $m \in \mathbb{N}$, the sequence $\pi_{n, m}^{\beta, f}$ converges to $f \mu$-maximally with respect to the $m$-meromorphy.

Theorem A generalizes Saff's theorem of Montessus de Ballore's type about multipoint Padé approximants (see [3]).

We now utilize the normalization of the polynomials $Q_{n, m}(z)$ with respect to a given open set $D_{m, \mu}$, that is,

$$
Q_{n, m}(z)=\prod\left(z-\alpha_{n, k}^{\prime}\right) \prod\left(1-z / \alpha_{n, k}^{\prime \prime}\right),
$$

where $\alpha_{n, k}^{\prime}, \alpha_{n, k}^{\prime \prime}$ are the zeros lying inside, resp. outside $D_{m, \mu}$. Under this normalization, for every compact set $K$ and $n$ large enough there holds

$$
\left\|Q_{n, m}^{\beta, f}\right\|_{K} \leq C_{1},
$$

where $C_{1}=C_{1}(K)$ is a positive constant, depending on $K$. In the sequel, we denote by $C_{i}$ positive constant, independent on $n$ and different at different occurrences.

In [8], the set $K_{\varepsilon}$ (look at the definition of a $\mu$-maximal convergence) is explicitly written, namely 
$K_{\varepsilon}:=K \backslash \Omega(\varepsilon)$, where

$$
\Omega(\varepsilon):=\bigcup_{n=1}^{\infty}\left(\bigcup_{\alpha_{n, k}^{\prime}}\left\{z,\left|z-\alpha_{n, k}^{\prime}\right|<\varepsilon /\left(2 m n^{2}\right)\right\}\right) .
$$

For $\Omega(\varepsilon)$ we have

$$
m_{1}(\Omega(\varepsilon)) \leq \varepsilon
$$

For points $z \notin \Omega(\varepsilon)$, we have

$$
\left|Q_{n, m}^{\beta, f}(z)\right| \geq C_{2}\left(\varepsilon / m n^{2}\right)^{k_{n}},
$$

where $k_{n}$ stands for the number of the zeros of $Q_{n, m}^{\beta, f}$ in $D_{m, \mu} ; k_{n} \leq m$.

Let $Q$ be the monic polynomial, the zeros of which coincide with the poles of $f$ in $D_{m, \mu} ; \operatorname{deg} Q \leq m$. It was proved in [8] (Proof of Lemma 2.3) that for every compact subset $K$ of $D_{m, \mu}$

$$
\limsup _{n \rightarrow \infty}\left\|f Q Q_{n, m}^{\beta, f}-Q P_{n, m}^{\beta, f}\right\|_{K}^{1 / n} \leq \frac{\left\|\mathrm{e}^{-U^{\mu}}\right\|_{K}}{R_{m, \mu}} .
$$

Hence, $-U^{\mu}(z)-\ln R_{m, \mu}$ is a harmonic majorant in $D_{m, \mu}$ of the family $\left\{\left|\left(f Q Q_{n, m}^{\beta, f}-Q P_{n, m}^{\beta, f}\right)(z)\right|^{1 / n}\right\}_{n=1}^{\infty}$.

Theorem B [8]: With $E, \mu, m, \omega_{n}$ and $f$ as in Theorem $A$, assume that $K$ is a regular compact set for which $\left\|\mathrm{e}^{-U^{\mu}}\right\|_{K}$ is not attained at a point on $E$. Suppose that the function $f$ is defined on $K$ and satisfies

$$
\underset{n \rightarrow \infty}{\limsup }\left\|f-\pi_{n, m}^{\beta, f}\right\|_{K}^{1 / n} \leq\left\|\mathrm{e}^{-U^{\mu}}\right\|_{K} / R<1
$$

Then $R \leq R_{m, \mu}(f)$.

Suppose that $\infty>R_{m, \mu}>\varrho_{\max }$ and $D_{m, \mu}$ is connected. Let $V$ be a disk in $D_{m} \backslash E_{\mu}\left(\varrho_{\max }\right)$, centered at a point $z_{0}$ of radius $r>0$ and such that $f$ is analytic on $V$. Fix $r_{1}, \quad 0<r_{1}<r$ and set $A:=\left\{z, r_{1} \leq\left|z-z_{0}\right| \leq r\right\}$. Fix a number $\varepsilon<\left(r-r_{1}\right) / 4$. Introduce, as before, the set $\Omega(\varepsilon)$. Recall that

$$
m_{1}(\Omega(\varepsilon)) \leq \varepsilon .
$$

It is clear that the set $A \backslash \Omega(\varepsilon)$ contains a concentric circle $\Gamma$ (otherwise we would obtain a contradiction with $m_{1}(\Omega(\varepsilon))<\left(r-r_{1}\right) / 4$.) We note that the function $f$ and the rational functions $\pi_{n, m}^{\beta, f}$ are well defined on $\Gamma$. Viewing (3), we may write

$$
\underset{n \rightarrow \infty}{\limsup }\left\|Q Q_{n, m}^{\beta, f} f-Q P_{n, m}^{\beta, f}\right\|_{\Gamma}^{1 / N} \leq\left\|\mathrm{e}^{-U^{\mu}}\right\|_{\Gamma} / R_{m, \mu},
$$

Suppose that

$$
\underset{n \rightarrow \infty}{\limsup }\left\|Q Q_{n, m}^{\beta, f} f-Q P_{n, m}^{\beta, f}\right\|_{\Gamma}^{1 / n}<\left\|\mathrm{e}^{-U^{\mu}}\right\|_{\Gamma} / R_{m, \mu} .
$$

or, what is the same,

$$
\underset{n \rightarrow \infty}{\limsup }\left\|Q Q_{n, m}^{\beta, f} f-Q P_{n, m}^{\beta, f}\right\|_{\Gamma}^{1 / n} \leq\left\|\mathrm{e}^{-U^{\mu}}\right\|_{\Gamma} /\left(R_{m, \mu}+\sigma\right)<1 .
$$

for an appropriate $\sigma>0$. Then,

$$
\left|\left(f-\pi_{n, m}^{\beta, f}\right)(z)\right|_{\Gamma} \leq C_{3}\left(n^{2} m / \varepsilon\right)^{m}\left(\left\|\mathrm{e}^{-U^{\mu}}\right\|_{\Gamma} /\left(R_{m, \mu}+\sigma\right)\right)^{n} .
$$

for all $z \in \Gamma$ and $n$ large enough. This leads to

$$
\underset{n \rightarrow \infty}{\limsup }\left\|f-\pi_{n, m}^{\beta, f}\right\|_{\Gamma}^{1 / n} \leq\left\|\mathrm{e}^{-U^{\mu}}\right\|_{\Gamma} /\left(R_{m, \mu}+\sigma\right) .
$$


using Theorem B, we arrive at $R_{m, \mu}+\sigma<R_{m, \mu}$. The contradiction yields

$$
\limsup _{n \rightarrow \infty}\left\|Q Q_{n, m}^{\beta, f} f-Q P_{n, m}^{\beta, f}\right\|_{\bar{V}_{\Gamma}}^{1 / n}=\left\|\mathrm{e}^{-U^{\mu}}\right\|_{\bar{V}_{\Gamma}} / R_{m, \mu},
$$

where $V_{\Gamma}$ is the disk bounded by $\Gamma$.

Then the function $-U^{\mu}-\ln R_{m, \mu}$ is an exact harmonic majorant of the family $\left\{\left|f Q Q_{n, m}^{\beta, f}-Q P_{n, m}^{\beta, f}\right|^{1 / n}\right\}$ in

$D_{m, \mu}$ (see (3)). Therefore, there exists a subsequence $\Lambda$ such that for every compact subset $K \subset D_{m, \mu} \backslash E$

$$
\lim _{n \rightarrow \infty, n \in \Lambda}\left\|Q f Q_{n, m}^{\beta, f}-P_{n, m}^{\beta, f} Q\right\|_{K}^{1 / n}=\left\|\mathrm{e}^{-U^{\mu}}\right\|_{K} / R_{m, \mu} .
$$

(see [9] [10]) for a discussion of exact harmonic majorant)). We will refer to this sequences as to an exact $\mu$ maximal convergent sequence to $f$ with respect to the $m$-meromorphy.

It is clear that for any $\varepsilon>o$ and each compactum $K \subset D_{m, \mu}$ there exists a set $K_{\varepsilon} \subset K$ such that $m_{1}\left(K \backslash K_{\varepsilon}\right)<\varepsilon$ and

$$
\lim _{n \rightarrow \infty, n \in \Lambda}\left\|f-\pi_{n, m}^{\beta, f}\right\|_{K \backslash K_{\varepsilon}}^{1 / n}=\left\|\mathrm{e}^{-U^{\mu}}\right\|_{\Gamma} /\left(R_{m, \mu}\right) .
$$

\section{Main Results and Proofs}

The main result of the present paper is

Theorem 1: Under the same conditions on $E$, assume that $\mu \in \mathcal{B}(\partial E)$ and that $\beta \subset \partial E$ is a triangular set of points. Let $m \in \mathbb{N}$ be fixed, $f \in \mathcal{A}(E)$ and $\varrho_{\max }<R_{m, \mu}<\infty$. Suppose that $D_{m, \mu}$ is connected. If for a subsequence $\Lambda$ of the multipoint Padé approximants $\pi_{n, m}^{\beta, f}$ condition (4) holds, then $\mu_{\omega_{n}} \rightarrow \mu$ as $n \rightarrow \infty$, $n \in \Lambda$.

The problem of the distribution of the points of interpolation of multipoint Padé approximants has been investigated, so far, only for the case when the measure $\mu$ coincides with the equilibrium measure $\mu_{E}$ of the compact set $E$. It was first raised by Walsh ([11], Chp. 3) while considering maximally convergent polynomials with respect to the equilibrium measure. He showed that the sequence $\mu_{\omega_{n}}$ converged weakly to $\mu_{E}$ through the entire set $\mathbb{N}$ (respectively their associated balayage measures onto the boundary of $E$ ) iff the interpolating polynomials at the points of $\beta$ of every function $f_{t}(z)$ of the form $f_{t}(z):=1 /(t-z), t$-fixed, $t \notin E$, converged $\mu_{E}$-maximally to $f_{t}$. Walsh's result was extended to multipoint Padé approximants with a fixed number of the free poles by Ikonomov in [12], as well as to generalized Padé approximants, associated with a regular condenser [13]. The case of polynomial interpolation of an arbitrary function $f \in \mathcal{A}(E)$ was considered by Grothmann [14]; he established the existence of an appropriate sequence $\Lambda$ such that $\mu_{\omega_{n}} \rightarrow \mu_{E}$, $n \rightarrow \infty, n \in \Lambda$, respectively the balayage measures onto $\partial E$. Grothmann's result was extended to multipoint Padé approximants $\pi_{n, m}^{\beta, f}$ with a fixed number of the free poles (see [15]). Finally, in [16] the case was considered, when the degrees of the denominators tended slowly to infinity, namely, $m_{n}=o(n / \ln n), n \rightarrow \infty$.

As a consequence of Theorem 1, we derive

Theorem 2: Under the conditions of Theorem 1, suppose that the $\mu$-exact maximally convergent sequence $\Lambda:=\left\{n_{k}\right\}_{k=1}^{\infty}$ satisfies the condition to be "dense enough", that is

$$
\limsup _{n_{k} \rightarrow \infty, n_{k} \in \Lambda} \frac{n_{k+1}}{n_{k}}<\infty .
$$

Then, there is at least one point $z_{0} \in \partial D_{m, \mu}(f)$ such that

$$
\limsup _{n \rightarrow \infty, n \in \Lambda} \mu_{P_{n, m}^{\beta, f}}\left(V_{z_{0}}(r)\right)>0 \text {. }
$$

Proof of Theorem 1: Set $Q_{n, m}^{\beta, f}:=Q_{n}, P_{n, m}^{\beta, f}:=P_{n}$ and $F:=f Q$. Fix numbers $R, \tau, r$ such that $\varrho_{\max }<R<\tau<r<R_{m, \mu}$ and $E_{\mu}(R)$ is connected. Then, by the conditions of the theorem, for every compactum $K \subset D_{m, \mu}$ (comp. (4))

$$
\lim _{n \in \Lambda}\left\|F Q_{n}-Q P_{n}\right\|_{K}^{1 / n}=\left\|\mathrm{e}^{-U^{\mu}}\right\|_{K} / R_{m, \mu}, \quad n \in \Lambda .
$$

Select a positive number $\eta$ such that $R+\eta<\tau<\tau+\eta<r<R_{m, \mu}$. Let $\Gamma$ be an analytic curve in 
$E_{\mu}(r) \backslash E_{\mu}(\tau+\eta)$ such that $\Gamma$ winds around every point in $E_{\mu}(\tau)$ exactly once. In an analogous way, we select a curve $\gamma \subset E_{\mu}(R+\eta) \backslash E_{\mu}(R)$. Additionally, we require that $U^{\mu}$ is constant on $\Gamma$ and $\gamma$. Set

$$
F_{n}(z):=\frac{1}{n} \ln \left|F Q_{n}-P_{n} Q\right|(z)+U^{\mu}(z)+\ln R_{m, \mu}, \quad n \in \Lambda .
$$

Let $\sigma>0$ be arbitrary. The functions $F_{n}$ are subharmonic in $E_{\mu}(r) \backslash E_{\mu}(R)$. By (5) and the choice of $\Gamma$,

$$
\max _{t \in \Gamma} F_{n}(t) \leq-\min _{t \in \Gamma}+\max _{t \in \Gamma}+\sigma \leq \sigma, \quad N \in \Lambda, \quad n \geq n_{1} 0 n_{1}(\sigma),
$$

and, analogously,

$$
\max _{t \in \gamma} F_{n}(t) \leq-\min _{t \in \Gamma}+\max _{t \in \Gamma} \leq \sigma, \quad N \in \Lambda, n>n_{1} .
$$

Then, by the max-principle of subharmonic functions,

$$
\max _{z \in A_{\gamma}, \Gamma} F_{n}(z) \leq \sigma, \quad n \in \Lambda, n \geq n_{1}, \quad N \in \Lambda,
$$

where $A_{\gamma, \Gamma}$ is the "annulus", bounded by $\Gamma$ and $\gamma$.

On the other hand, by (5), there exists, for every compact set $K \subset E_{r} \backslash E_{R}$ and $n$ large enough, a point $z_{n, K} \in K$ such that

$$
-U^{\mu}\left(z_{n, K}\right)-\ln R_{m, \mu}-\sigma \leq \frac{1}{n} \ln \left|F Q_{n}\left(z_{n, K}\right)-Q P_{n}\left(z_{n, K}\right)\right|, \quad n \geq n_{3}(K), n \in \Lambda .
$$

Therefore,

$$
-\sigma \leq F_{n}\left(z_{n, K}\right), \quad n \geq n_{2}(K, \sigma) .
$$

Further, by the formula of Hermite-Lagrange, for $z \in \gamma$ we have

$$
F Q_{n}(z)-Q P_{n}(z)=\frac{1}{2 \pi i} \int_{\Gamma} \frac{\omega_{n+m+1}(z)}{\omega_{n+m+1}(t)} \frac{F Q_{n}(t)-Q P_{n}(t)}{t-z} \mathrm{~d} t .
$$

Hence, by (5),

$$
\begin{aligned}
& \frac{1}{n} \ln \left|F Q_{n}(z)-Q P_{n}(z)\right| \leq \max _{t \in \Gamma} U^{\omega_{n+m+1}}(t)-U^{\omega_{n+m+1}}(z)+\frac{1}{n} \ln \left\|F Q_{n}-Q P_{n}\right\|_{\Gamma}+\frac{1}{n} \text { const } \\
& \leq \max _{t \in \Gamma} U^{\omega_{n+m+1}}(t)-U^{\omega_{n+m+1}}(z)-\min _{t \in \Gamma} U^{\mu}(t)-\ln R_{m, \mu}+\sigma, \\
& n \in \Lambda, \quad n \geq n_{3}=n_{3}(\sigma)>n_{1},
\end{aligned}
$$

where $U^{\omega_{n+m+1}}:=U^{\mu_{\omega_{n+m+1}}}$. To simplify the notations, we set $U^{\omega_{n+m+1}}:=U^{\omega_{n}}$ (the correctness will be not lost, since $m \in \mathbb{N}$ is fixed). Involving into consideration the functions $F_{n}$ (see (6)), we get for $z \in \gamma$

$$
\begin{aligned}
F_{n}(z) \leq & \max _{t \in \Gamma}\left(U^{\omega_{n}}(t)-U^{\mu}(t)\right)+\max _{t \in \Gamma} U^{\mu}(t)+\left(U^{\mu}(z)-U^{\omega_{n}}(z)\right) . \\
& -\min U^{\mu}(t)+\sigma, \quad n \in \Lambda, \quad n \geq n_{2} \geq n_{1}
\end{aligned}
$$

By Helly's selection theorem [1], there exists a subsequence of $\Lambda$ which we denote again by $\Lambda$ such that $\mu_{\omega_{n+m+1}}:=\mu_{\omega_{n}} \rightarrow \omega, n \in \Lambda$. Passing to the limit, we obtain

$$
\underset{\Lambda}{\limsup }\left|F_{n}(z)\right| \leq \max _{t \in \Gamma}\left(U^{\omega}(t)-U^{\mu}(t)\right)+\left(U^{\mu}(z)-U^{\omega}(z)\right), \quad z \in \gamma .
$$

Consider the function $\phi$, harmonic in $A_{\Gamma, \gamma}$ and

$$
\phi:= \begin{cases}0, & \Gamma, \\ \min \left(0,-\min _{t \in \gamma}\left(U^{\mu}(t)-U^{\omega}(t)\right)+\left(U^{\mu}(z)-U^{\omega}(z)\right)\right), & \gamma .\end{cases}
$$


From (7) and (9), we arrive at

$$
\limsup F_{n}(z) \leq \phi,
$$

for $z$ in $A_{\Gamma, \gamma}$. Being harmonic, $\phi$ obeys the maximum and the minimum principles in this region. The definition yields

$$
\phi(z) \leq 0, \quad z \in A_{\Gamma, \gamma},
$$

We will show that

$$
\phi(z) \equiv 0
$$

Suppose that (10) is not true. Let $\Upsilon$ be a closed curve in the set $E_{R+\eta}-\gamma^{o}$, where $\gamma^{o}$ stands for the interior of $\gamma$. Then there exists a number $\theta>0$ such that $\phi \leq-\Theta$ for every $z \in \Upsilon$. This inequality contradicts (8), for $\sigma$ close enough to the zero and $n \in \Lambda$ sufficiently large.

Hence, $\phi \equiv 0$. Then the definition of $\phi$ yields

$$
U^{\mu}(z)-U^{\omega}(z) \equiv \min _{t \in \gamma}\left(U^{\mu}(t)-U^{\omega}(t)\right), \quad z \in \gamma .
$$

The function $U^{\mu}(z)-U^{\omega}(z)$ is harmonic in the unbounded complement $G$ of $\gamma$, and by the maximum principle,

$$
U^{\mu}(z)-U^{\omega}(z) \equiv \text { Constant, } \quad z \in G
$$

consequently,

$$
U^{\mu}(z)-U^{\omega}(z) \equiv \text { Constant }, \quad z \in E^{c} .
$$

On the other hand, $\left(U^{\mu}-U^{\omega}\right)(\infty)=0$, which yields $U^{\mu} \equiv U^{\omega}$ in $E^{c}$. By Carleson's Lemma, $\mu=\omega$. On this, Theorem 1 is proved.

The proof of Theorem 2 will be preceded by an auxiliary lemma

Lemma 1 [17]: Given a domain $U$, a regular compact subset $S$ and a sequence $\vartheta:=\left\{n_{k}\right\}$ of positive integers, $n_{k}<n_{k+1}, k=1,2, \cdots$, such that

$$
\limsup _{n_{k} \rightarrow \infty, n_{k} \in \Lambda} \frac{n_{k+1}}{n_{k}}<\infty,
$$

Suppose that $\left\{\phi_{n_{k}}\right\}$ is a sequence of rational functions, $\phi_{n_{k}} \in R_{n_{k}, n_{k}}, k-1,2, \cdots, \phi_{n_{k}}=\phi_{n_{k}}^{\prime} / \phi_{n_{k}}^{\prime \prime}$ having no more that $m$ poles in $U$ and converging uniformly of $\partial S$ to a function $\phi \neq 0$ such that

$$
\limsup _{n_{k} \rightarrow \infty, n_{k} \in \Lambda}\left\|\phi_{n_{k}}-\phi\right\|_{\partial S}^{1 / n}<1 .
$$

Assume, in addition, that on each compact subset of $U$

$$
\lim _{n_{k} \rightarrow \infty, n_{k} \in \Lambda} \mu_{\phi_{n_{k}}^{\prime}}(K)=0 .
$$

Then the function $\phi$ admits a continuation into $U$ as a meromorphic function with no more than $m$ poles.

Proof of Theorem 2: We preserve the notations from the proof of Theorem 1.

The proof of Theorem 2 follows from Lemma 1 and Theorem 1. Indeed, under the conditions of the theorem the sequence $\left\{\pi_{n}\right\}_{n \in \Lambda}$ converges maximally to $f$ with respect to the measure $\mu$ and the domain $D_{m, \mu}$. Hence, inside $D_{m, \mu}$ (on compact subsets) condition (11) if fulfilled. From the proof of Theorem 1, we see that there is a regular compact subset $S$ of $D_{m, \mu}$ such that $\limsup _{n \in \Delta}\left\|f-\pi_{n}\right\|_{S}^{1 / n}<1$.

Suppose now that the statement of Theorem 2 is not true. Then there is, for every $z \in \partial D_{m, \mu}$ a disk $V_{z}\left(r_{z}\right):=V_{z}, r_{z}>0$ with $\lim _{n_{k}} \mu_{P_{n}}\left(V_{z}\right)=0$. We select a finite covering of disks $V_{z_{j}}$ such that $W:=\bigcup V_{z_{j}} \supset \partial D_{m, \mu}$. Condition (11) holds inside $W$. Applying Lemma 1 with respect to the sequence $\pi_{n}$ and to the domain $D_{m, \mu} \bigcup W$, we conclude that $f \in \mathcal{M}_{m}\left(\overline{D_{m, \mu}}\right)$. This contradicts the definition of $D_{m, \mu}$.

On this, the proof of Theorem 2 is completed. 
Using again Lemma 1 and applying Theorem A, we obtain a more general result about the zero distribution of the sequence $\left\{\pi_{n, m}^{\beta, f}\right\}$.

Theorem 3: Let $E$ be a regular compactum in $\mathbb{C}$ with a connected complement, let $\mu \in \mathcal{B}(E)$ and $\beta \in E$ be a triangular point set. Let the polynomials $\omega_{n}, n=1,2, \cdots$, be defined as above. Suppose that $\mu_{\omega_{n}} \rightarrow \mu$ as $n \rightarrow \infty$ and $f \in \mathcal{A}(E)$. Let $m \in \mathbb{N}$ be fixed, and suppose that $R_{m, \mu}<\infty$. Then there is at least one point $z_{0} \in \partial D_{m, \mu}$ such that $\limsup _{n \rightarrow \infty} \mu_{\pi_{n, m}^{\beta, f}}\left(\bar{V}_{z_{0}}(r)\right)>0$ for every positive $r$.

\section{Acknowledgements}

The author is very thankful to Prof. E. B. Saff for the useful discussions.

\section{References}

[1] Saff, E.B. and Totik, V. (1997) Logarithmic Potentials with External Fields. Grundlehren der mathematischen Wissenschaften, 316. http://dx.doi.org/10.1007/978-3-662-03329-6

[2] Carleson, L. (1964) Mergelyan's Theorem on Uniform Polynomial Approximation. Mathematica Scandinavica, 15, 167-175.

[3] Saff, E.B. (1972) An Extension of Montessus de Ballore Theorem on the Convergence of Interpolation Rational Functions. Journal of Approximation Theory, 6, 63-67. http://dx.doi.org/10.1016/0021-9045(72)90081-0

[4] Kovacheva, R.K. (1989) Generalized Padé Approximants of Kakehashi's Type and Meromorphic Continuation of Functions. Deformation of Mathematical Structures, 151-159. http://dx.doi.org/10.1007/978-94-009-2643-1_14

[5] Perron, O. (1929) Die Lehre von den Kettenbrüchen. Teubner, Leipzig.

[6] Gonchar, A.A. (1975) On the Convergence of Generalized Padé Approximants of Meromorphic Functions. Matematicheskii Sbornik, 98, 564-577. English Translation in Mathematics of the USSR-Sbornik, 27, 503-514.

[7] Tsuji, M. (1959) Potential Theory in Modern Function Theory. Maruzen, Tokyo.

[8] Bello Hernándes, M. and De la Calli Ysern, B. (2013) Meromorphic Continuation of Functions and Arbitrary Distribution of Interpolation Points. Journal of Mathematical Analysis and Applications, 403, 107-119. http://dx.doi.org/10.1016/j.jmaa.2013.02.014

[9] Walsh, J.L. (1946) Overconvergence, Degree of Convergence, and Zeros of Sequences of Analytic Functions. Duke Mathematical Journal, 13, 195-234. http://dx.doi.org/10.1215/S0012-7094-46-01320-8

[10] Walsh, J.L. (1959) The Analogue for Maximally Convergent Polynomials of Jentzsch's Theorem. Duke Mathematical Journal, 26, 605-616. http://dx.doi.org/10.1215/S0012-7094-59-02658-4

[11] Walsh, J.L. (1969) Interpolation and Approximation by Rational Functions in the Complex Domain. Vol. 20, American Mathematical Society Colloquium Publications, New York.

[12] Ikonomov, N. (2013) Multipoint Padé Approximants and Uniform Distribution of Points. Comptes Rendus de l'Academie Bulgare des Sciences, 66, 1097-1105.

[13] Ikonomov, N. (2014) Generalized Padé Approximants for Plane Condenser. Mathematica Slovaca, Springer, Accepted for Publication in 2014.

[14] Grothmann, R. (1996) Distribution of Interpolation Points. Arkiv för matematik, 34, 103-117. http://dx.doi.org/10.1007/BF02559510

[15] Ikonomov, N. and Kovacheva, R.K. (2014) Distribution of Points of Interpolation of Multipoint Padé Approximants. AIP Conference Proceedings, AMEE2014, 1631, 292-296. http://dx.doi.org/10.1063/1.4902489

[16] Blatt, H.P. and Kovacheva, R.K. (2015) Distribution of Interpolation Points of Maximally Convergent Multipoint Padé Approximants. Journal of Approximation Theory, 191, 46-57.

[17] Kovacheva, R.K. (2010) Normal Families of Meromorphic Functions. Comptes Rendus de l'Academie Bulgare des Sciences, 63, 807-814. 